\title{
Inflammatory monocytes-a novel therapeutic target for ALS?
}

In a mouse model of amyotrophic lateral sclerosis (ALS), neuropathology occurs following recruitment of inflammatory monocytes to the spinal cord, and therapeutic targeting of these cells can reduce neuronal loss and prolong survival, according to a recent study. The finding that such monocytes are also present in patients with ALS suggests targeting of inflammatory monocytes as a novel therapeutic approach to this disease.

"It has been known for a number of years that there is a 'microgliosis' in the CNS of animals with the SOD1 mutation [a model of ALS] and in patients with ALS," says Howard Weiner, lead researcher of the study, "but no one knew what these cells were." After identifying markers of resident microglia and recruited proinflammatory monocytes $\left(\mathrm{CD} 39^{+}\right.$and Ly6C $\mathrm{C}^{\text {hi }}$, respectively) to distinguish these two cell types, the researchers performed gene and microRNA profiling to characterize these cell populations at various stages of disease in SOD1-mutant mice.

Prior to disease onset, Ly6C $\mathrm{C}^{\text {hi }}$ monocytes in the spleen had a proinflammatory phenotype. Over the course of disease, the proportion of Ly6 $\mathrm{C}^{\text {hi }}$ monocytes in the spinal cord increased from 1-2\% to $31 \%$, which was accompanied by a decrease in the proportion of resident

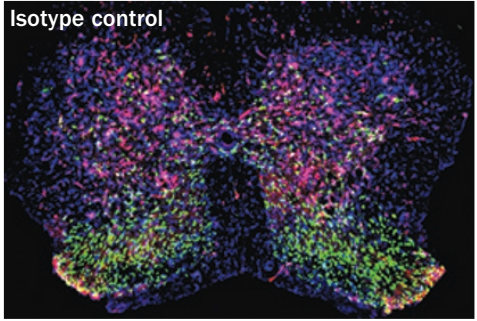

$\mathrm{CD} 9^{+}$microglia. Notably, no influx of the proinflammatory monocyte population was evident in the brain.

At disease onset, $\mathrm{CD} 39^{+}$microglia from the spinal cord upregulated expression of $\mathrm{Ccl} 2$, which encodes C-C motif chemokine 2 (CCL2), and Ly6C ${ }^{\text {hi }}$ cells showed increased expression of $\mathrm{Ccr} 2$, which encodes the cognate receptor of CCL2. Further investigation revealed that spinal cord microglia directly recruited inflammatory monocytes to this site.

To determine whether the recruited monocytes were involved in disease progression, SOD1-mutant mice were treated with anti-Ly6C monoclonal antibody. "Modulating the Ly6C hi cells decreased their infiltration into the CNS, modulated their inflammatory signature, protected neurons from damage, and prolonged survival," explains Weiner.

In patients with ALS, peripheral blood $\mathrm{CD} 14^{+} \mathrm{CD} 16^{-}$cells-which are

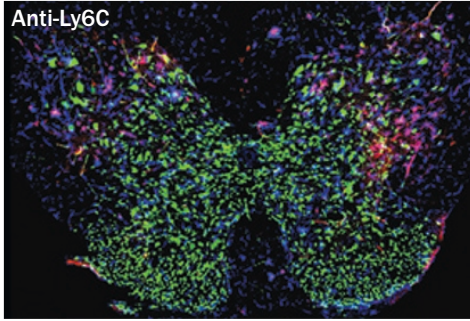

Treatment with antiLy6C monoclonal antibody decreases infiltration of inflammatory monocytes (red) into the spinal cord and attenuates loss of neurons (green) in ALS mice. Image courtesy of O. Butovsky. analogous to the $\mathrm{Ly} 6 \mathrm{C}^{\mathrm{hi}}$ population - had a proinflammatory microRNA profile similar to that seen in the SOD1mutant mice. Furthermore, high levels of CCL2 were detected in cerebrospinal fluid samples from patients with ALS. Importantly, these findings indicate that the disease process observed in the mouse model is similar to that in human ALS.

Weiner and colleagues aim to further study the microRNA signature of monocytes in ALS, and plan to perform clinical trials of interventions that target inflammatory monocytes in individuals with this disease.

\section{Katy Malpass}

Original article Butovsky, 0. et al. Modulating inflammatory monocytes with a unique microRNA gene signature ameliorates murine ALS. J. Clin. Invest. doi:10.1172/ JCl62636 